\title{
3D Printed Patient-Specific Acetabular Jig for Cup Placement in Total Hip Arthroplasty
}

\author{
Abhishek Mishra ${ }^{1,3} \cdot$ Tarun Verma $^{1} \cdot$ Rajkumar $^{1} \cdot$ Gaurang Agarwal $^{1} \cdot$ Amit Sharma $^{2} \cdot$ Lalit Maini $^{1}$
}

Received: 25 August 2019 / Accepted: 29 October 2019 / Published online: 17 March 2020

(c) Indian Orthopaedics Association 2020

\begin{abstract}
Introduction Acetabular cup placement is an important modifiable factor determining complication rates like aseptic loosening and hip dislocation related to faulty cup placement, which by standard method is largely dependent upon eyeballing and surgeon's judgment. We evaluated a self-designed, low-cost, patient-specific acetabular jig to guide cup placement in total hip arthroplasty in comparison to conventional technique.

Methods It was a prospective randomized control study. Thirty-six patients were categorized into group-A \& group-B. In group-A, virtually designed acetabular jig was 3 Dimensional (3D) printed and used intra-operatively to guide cup placement. In group-B, the standard method of cup placement was used. Acetabular cup placement was evaluated on postoperative $\mathrm{x}$-rays and compared between two groups.

Results In group-A, angle of anteversion were significantly in centre of range of safe zone as compared to group $\mathrm{B}$ in which hip is maximally stable with more precision in creating hip centre as compared to group-B without any significant $(p=0.325)$ increase in surgical time or blood loss.

Conclusion Computed tomography (CT) scan based virtual pre-operative templating and cup placement guided by virtually designed, patient-specific acetabular jig is a low-cost tool with a short learning curve which can be designed and made available easily. It is a useful tool in decreasing chances of malpositioning of cup and recreates hip centre close to anatomical one especially in cases where anatomy has been distorted such as bony ankylosis and developmental dysplasia of hip.
\end{abstract}

Keywords 3D Printing $\cdot$ Acetabular cup $\cdot$ Acetabular jig $\cdot$ Patient-specific instrumentation $\cdot$ X-ray Templating

\section{Introduction}

Abhishek Mishra

mishra.abhishek85@gmail.com

Tarun Verma

tarunamiabledpv@gmail.com

Rajkumar

Rajkumargautam12@gmail.com

Gaurang Agarwal

gaurang140389@gmail.com

Amit Sharma

dramite31@gmail.com

Lalit Maini

lalit_maini@rediffmail.com

1 Maulana Azad Medical College and Lok Nayak Hospital, New Delhi, India

2 Lady Hardinge Medical College, New Delhi, India

3 Plot no. 60, Vivek Nagar Colony, Nasirpur, Varanasi, Uttar Pradesh, India
Aseptic loosening and hip dislocation remain two of the main complications of total hip arthroplasty (THA). Positioning of acetabular component is a central factor for preventing these complications $[1,2]$. Suboptimal placement of the acetabular component in THA can lead to hip instability, impingement, increased wear and reduced range of motion [3]. The conventional methods of using pre-operative radiographs for pre-operative planning and standard surgical instrumentation are not very accurate and depend on surgeon's experience [4]. In complex primary THA like malunited acetabulum fracture and ankylosed hip, the cup size and placement in correct orientation can be challenging.

Computer-assisted surgery (CAS) techniques are evolving to make surgical procedures more accurate. Although CAS systems improve the accuracy of cup placement [5], they are having drawbacks such as increased operative time, higher cost, increased complexity of the procedure, 
need to change patient's position during the procedure and difficult referencing. Recently, rapid prototyped patientspecific guides have been created for cup placement designed from pre-operative computed tomography (CT) scans. Designing patient-specific instrumentation based on patient's unique bone morphology from CT scan is an improvement over generic instruments by minimizing errors from standard surgical instruments that depend on patient positioning, pelvic orientation, surgical exposure and surgeon's experience [6, 7]. However, they require removing more soft tissue around acetabular rim, which add to the surgical time and still have challenges to achieve good accuracy.

Our objective was to study design characteristics and accuracy of patient-specific acetabular jig for guiding cup placement.

\section{Materials and Methods}

This prospective randomized control study was conducted on patients with various hip disorders, who presented to our institute from October 2015 to March 2017. All adult patients indicated for total hip replacement were included in this study with patients having any metallic implant in the vicinity of operative hip distorting pre-operative CT scan images and revision total hip arthroplasty were kept under exclusion criteria. Thirty-six hips satisfying the following inclusion/exclusion criteria were randomized into two groups; 18 each in Group-A (Cases) and Group-B (Control group) using computer randomization software. In GroupA, CT based virtual surgical planning to design patient-specific acetabular jigs was done and 3D printed jigs were used to guide cup placement during surgeries, while in Group$\mathrm{B}$, conventional method of templating on $\mathrm{X}$ rays and cup placement was used. Informed consent was obtained from all the participants in the study. Ethical clearance was taken from institute ethical committee prior to the beginning of this study with institutional review board (IRB) clearance number F.NO./11/IEC/MAMC/2015/317.

All patients were operated under regional anesthesia in lateral position by the posterior approach to hip operated by a single surgeon. A pre-operative dose of tranexamic acid was given according to the weight of the patients.

\section{Methodology}

Antero-posterior radiograph of bilateral hip with thighs of each case was done in both groups for $\mathrm{x}$-ray based templating. The simplified methodology is depicted in the flowchart (Fig. 1).

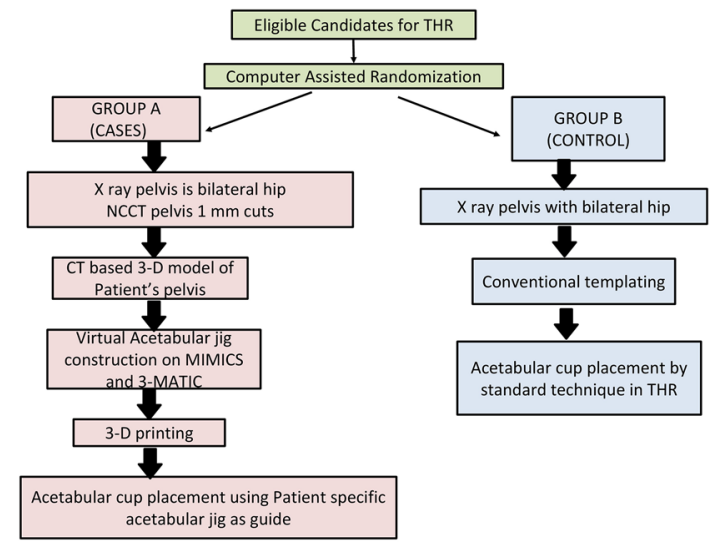

Fig. 1 Flowchart of methodology

\section{Group-A}

The workflow in Group A is shown in Figs. 2, 3, 4 and 5. All the patients in Group A had undergone NCCT (Noncontrast computed tomography) scan of pelvis with bilateral hip with $1 \mathrm{~mm}$ cuts and DICOM (Digital Imaging and Communication in Medicine) images obtained were imported in Mimics software (version 17.0). A virtual 3D model of the pelvis with subtracted femur bones was generated, which was imported in 3-matic software (version 9.0) as Stereolithography (STL) file. Contralateral unaffected acetabular cavity was marked using "brush mark" command. A fitting sphere based on marked acetabular surface was made using "create analytical primitive" and "convert analytical primitive to part" commands. The diameter of this sphere represented the cup size. A second sphere of $5 \mathrm{~mm}$ larger diameter than the previous sphere was made surrounding the 1 st sphere. The second sphere was made hollow using "hollow" command with $5 \mathrm{~mm}$ rim. It was then trimmed into a ring of $5 \mathrm{~mm}$ width which surrounded the acetabular rim. The ring was mirrored to affected side by "mirror" command using mid-plane as reference. The orientation of the ring was checked with respect to inclination and anteversion. Antero-inferior part of the ring was trimmed off with reference to the posterior end of the transverse acetabular ligament (TAL).

An attachment structure (small contoured plate) was designed over postero-superior aspect of acetabular rim using "create curve" and "design plate" commands. This was merged to the ring by "Boolean Union" command to complete the acetabular jig. Holes of $2 \mathrm{~mm}$ diameter were made in the attachment structure for $\mathrm{k}$ wire fixation of the jig (when used intra-operatively). The virtual jig was then exported as STL file and 3D printed in polylactic acid (PLA) material. The 3D printed jig was autoclaved and used as a guiding tool for cup placement. Acetabular osteophytes have to be removed and jig was used after removal of osteophytes. 
Fig. 2 Creation of 3D model of pelvis from NCCT pelvis and marking of contralateral acetabular cavity
Fig. 3 Designing of acetabular jig using marked acetabulum
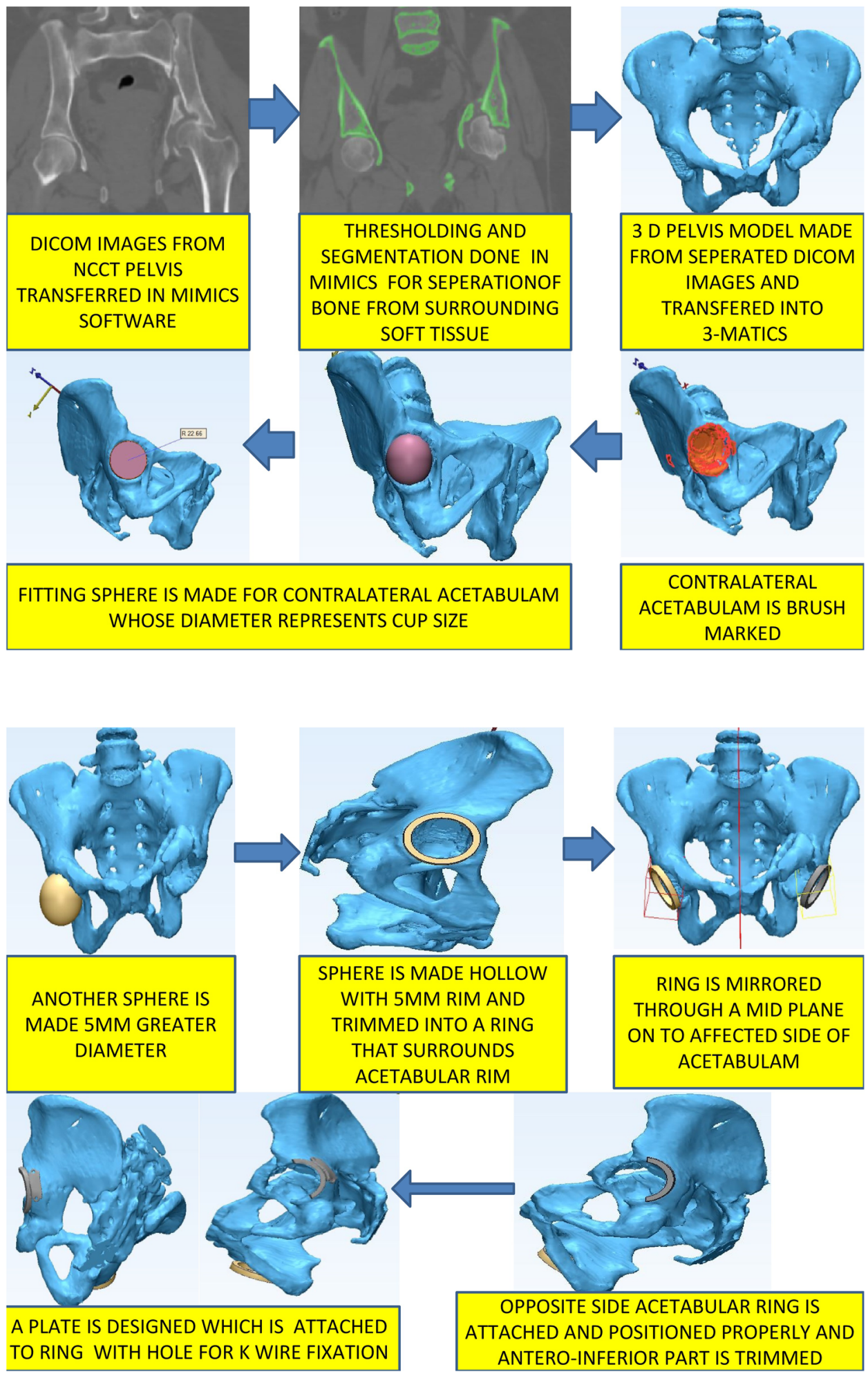

Jig is placed over postero superior aspect of acetabulum with the help of a plate attached to the jig through $\mathrm{k}$ wires. Virtual pre-operative planning is done accordingly that plate matches the contour over the posterosuperior aspect of acetabulum and is placed over the desired area fitting the contour and fixed with $\mathrm{k}$ wires after removing osteophytes of acetabulum. After its proper seating, ring part of jig surrounds posterosuperior rim of acetabulum for guiding of cup placement. Then acetabulum was reamed and acetabular cup was placed using the jig as a guide for orientation. 


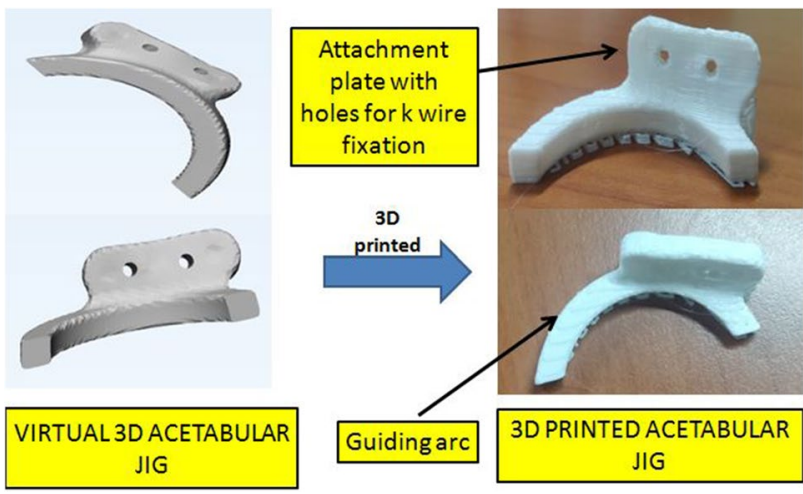

Fig. 4 3D printing of acetabular jig

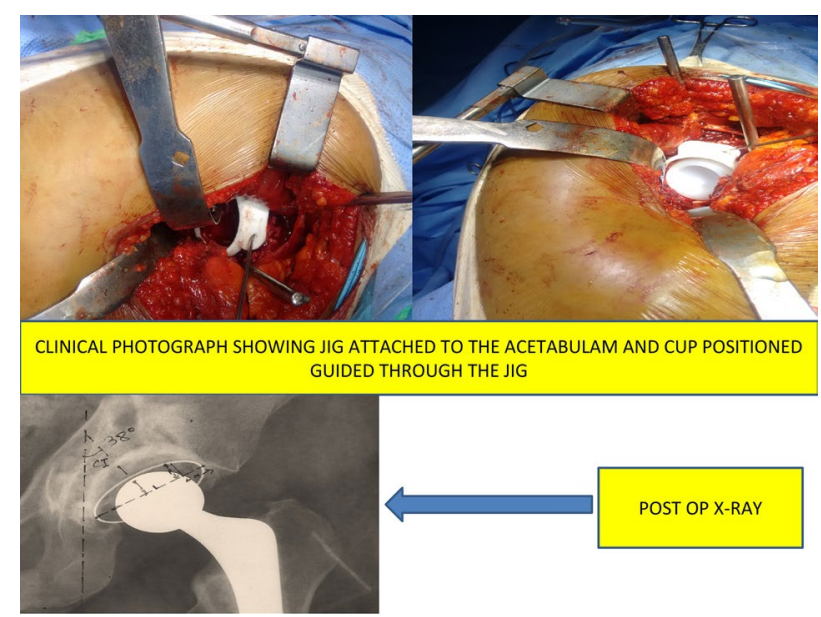

Fig. 5 Using acetabular jig intra-operatively for guiding cup placement

Required anteversion and inclination is achieved using jig as a reference.

\section{Group-B}

Conventional total hip arthroplasty protocol was followed. Pre-operative X-ray based templating was done to decide the component size. Placement of Acetabular cup was based on conventional/standard instrumentation and surgeon's judgment.

Post-operative antero-posterior radiograph of pelvis with bilateral hip in $100 \%$ magnification was done in both the groups for assessment.

A comparison was done between the two groups on surgical duration, surgical time for cup placement and blood loss intra-operatively. Post-operatively inclination and version of acetabular cup were assessed on post-operative radiograph. The position of hip centre on the replaced side was compared with the unaffected side in both groups by measuring acetabular offset and hip length and the difference was measured [8].

Angle of anteversion was calculated using the formula given by Pradhan et al. [9]. Also, notes were made regarding difficulties faced in placing the jig during surgeries as well as design modifications required.

Statistical tests were performed using statistical package for social sciences SPSS 21.0 software.

Analysis of all parameters was done after evaluating whether the data was normally distributed or not. Normality of data was tested by Kolmogorov-smirnov test. Data in which assumptions were fulfilled, we have used independent $t$ test for comparison of quantitative variables between two groups. Data in which assumptions were not fulfilled, we used Mann-Whitney $U$ test for comparison. Chi-square test has been used for comparing parameters in which outcome/ data is ordinal i.e. not continuous (qualitative data). A $p$ value less than 0.05 was considered statistically significant.

\section{Observation and Results}

From October 2015 to March 2017, thirty-six patients had undergone total hip replacement under two groups (A and B) and following data (Table 1) were collected, evaluated and assessed. Design characteristics of acetabular jig evolved during our study with many variations and we arrived at a design characteristic for acetabular jig which should be a one-third ring over postero-superior aspect of acetabulum rim of $5 \mathrm{~mm}$ thickness for guiding cup placement. That ring has an attachment part made of a plate with $1 \mathrm{~cm}$ thickness
Table 1 Table of summary of findings in both case and control group

\begin{tabular}{llll}
\hline S no. & Average value of parameters evaluated in our study & Case group & Control group \\
\hline 1 & Blood loss & $519.44 \mathrm{ml}$ & $495.56 \mathrm{ml}$ \\
2 & Surgical duration & $99.39 \mathrm{~min}$ & $92.33 \mathrm{~min}$ \\
3 & Surgical duration of cup placement & $55.22 \mathrm{~min}$ & $51.56 \mathrm{~min}$ \\
4 & Angle of inclination & $43.28^{\circ}$ & $44.11^{\circ}$ \\
5 & Angle of anteversion & $14.22^{\circ}$ & $13.42^{\circ}$ \\
6 & Difference of caetabular offset between normal and replaced hip & 0.0222 & 0.0278 \\
7 & Difference of hip length between normal and replaced hip & 0.0167 & 0.15 \\
\hline
\end{tabular}


matching contour of postero superior surface adjacent to acetabulum where it is seated and fixed with two $\mathrm{k}$ wires through the holes created in plate for $\mathrm{k}$ wire fixation.

Surgical duration in Group-A and Group-B with mean values was 99.4 and $92.3 \mathrm{~min}$, respectively. Total blood loss in Group-A and Group-B with mean values was $519 \mathrm{ml}$ and $496 \mathrm{ml}$, respectively. Mean surgical time for cup placement in Group-A and Group-B was $55 \mathrm{~min}$ and $52 \mathrm{~min}$, respectively. The surgical duration and blood loss were more in Group-A than Group-B, but the difference was found to be statistically insignificant indicating that acetabular jig technique does not increase surgical invasiveness and blood loss significantly. Average angle of inclination measured on post-operative X-ray in Group-A and Group-B was $43.3^{\circ}$ (range: $38^{\circ}-46^{\circ}$ ) and $44^{\circ}$ (range: $34^{\circ}-50^{\circ}$ ), respectively, with a difference of $0.83 \pm 1.64$. Average angle of anteversion calculated from post-operative X-ray in GroupA and Group-B was $14.2^{\circ}$ (range: $8^{\circ}-27^{\circ}$ ) and $13.4^{\circ}$ (range: $\left.5^{\circ}-36^{\circ}\right)$, respectively, with a difference of $0.8 \pm 3.3$. The angle of anteversion in Group-A were more in the centre of the safe range with less variability and outliers than GroupB. Coefficient of variation in Group A is 0.327 as compared to 0.593 in Group B.

Difference in acetabular offset between replaced hip and contralateral hip was evaluated and compared between cases and control as an indicator of accuracy in creating hip centre as compared to contralateral normal hip centre. Mean difference in Group-A and Group-B came out to be $0.022 \mathrm{~mm}$ and $0.028 \mathrm{~mm}$, respectively. Mean difference in GroupA and Group-B came out to be $0.017 \mathrm{~mm}$ and $0.15 \mathrm{~mm}$, respectively. Although center of rotation in group A was more accurately close to the original hip center as compared to group B, difference was not significant probably due to small sample size.

The size of cup used intra-operatively matched the planned size on CT based 3D model in all the 18 hips in Group-A, while in Group-B, discrepancy between size determined from $\mathrm{x}$-ray based templating and actual cup placed was found in 6 out of 18 hips.

\section{Discussion}

Premature implant failure in total hip arthroplasty requiring revision is a limitation, often resulting from poor implant placement $[4,7,10-14]$. The inclination and anteversion of the acetabular component, and the anteversion and the offset of the femoral component are important for longevity in THA because malposition of the components can lead to post-operative limitation of activities of daily living, dislocation wear issues at the articulation, and aseptic loosening $[11,15]$. In our study we evaluated accuracy of actebular cup placement guided by $3 \mathrm{D}$ printed jig and got an average angle of inclination measured on post-operative $\mathrm{x}$-ray in Group A and Group-B was $43.3^{\circ}$ and $44^{\circ}$, respectively, and average angle of anteversion calculated from post-operative x-ray in Group-A and Group-B was $14.2^{\circ}$ and $13.4^{\circ}$, respectively, with the difference of cup placement being $0.83^{\circ} \pm 1.64^{\circ}$ for inclination and $0.8^{\circ} \pm 3.3^{\circ}$ for anteversion. James et al. studied the accuracy of acetabular component using computer navigation and found out that the accuracy of cup placement (mean \pm standard deviation of the absolute difference between computer-assisted navigation and CT) was $1.8^{\circ} \pm 1.2^{\circ}$ for inclination and $2.0^{\circ} \pm 2.0^{\circ}$ for anteversion [16]. Ernst et al. did a similar study for evaluation of cup placement by computer navigation assisted technique and found an average inclination of $42.3^{\circ}$ and an average anteversion of $24.5^{\circ}$ in the computer-assisted study group and an average inclination of $37.9^{\circ}$ and an average anteversion of $23.8^{\circ}$ in the freehand group [17]. Although the accuracy of cup placement increased significantly with the above methods, cost is also increased significantly with the use of this technique. Xiau et al. used iPhone technology in improving acetabular component position in total hip arthroplasty and found the difference in inclination angles using iPhone application compared with the freehand method to be statistically significant in the "untrained group" suggesting a potential role for iPhone applications in junior surgeons in overcoming the steep learning curve [18]. Nicholas et al. studied positioning of acetabular component using anatomical landmarks (Ilium, superior pubic ramus and superior acetabulum) which used to define a plane allowing orientation of cup in abduction and anteversion on 24 cadaveric acetabulum and 617 primary total hip arthroplasties performed between 1996 and 2003 but it had limitation of being very little useful in dysplastic hips or post acetabular fracture where anatomical landmarks have been distorted [19]. Preoperative planning by reviewing the clinical and radiographic findings of a patient before surgery is standard of care in joint arthroplasty guiding a surgeon through the entire procedure while achieving optimal and reproducible results. Currently, implant placement in THA relies on navigation, crude instrumentation, or exclusively on a surgeon's intraoperative assessment of limited anatomic landmarks with the use of generic, mechanical instruments designed for all patients with differing severities of pathology and unique anatomy [20]. The most widely used method of placing a cup involves planning the surgery with preoperative radiographs and attempting to reproduce the plan with standard surgical instruments which fail to account for a patient's unique pelvic anatomy and position on the operating table (OT). Transferring the plan from the radiographs to the OT can be difficult due to a patient's unique anatomy and the differences defined by Murray between radiographic, operative, and anatomic planes of version and abduction [21]. Surgeon experience using an alignment guide attached to a reamer 
handle is used to obtain $45^{\circ}$ abduction referencing the floor and $20^{\circ}$ anteversion referencing the longitudinal axis of the patient. Assessing the position of the pelvis relative to the patient and the OT using standard instruments can lead to malpositioned acetabular components [20]. For acetabular components, the safe zone for orientation is $15^{\circ} \pm 10^{\circ}$ of anteversion and $40^{\circ} \pm 10^{\circ}$ of inclination [3]. Accurate acetabular cup positioning in THA decreases the risk of dislocation, impingement, and all component wear rate [20, $22,23]$. Defining 3D relationships at the time of surgery for acetabular bone preparation can be difficult, especially when there is significant bone deformity, even by most experienced surgeons.

Technologies including intra-operative computer navigation and preoperative computer-assisted surgical planning with the fabrication of patient-specific instruments (PSI) based on a patient's unique bony morphologic features of the acetabulum from a computed tomography (CT) scan is an improvement over generic instruments by minimizing sources of error from standard surgical instruments that depend on appropriate patient positioning, pelvis orientation, exposure, and surgeon experience and by accomplishing acetabular orientation in 3 dimensions improving bone preparation and component positioning [20]. But surgical navigation for THA has its own drawbacks like higher cost, increased duration; need to change patient position during surgery, difficult referencing and increased complexity of procedure.

Various authors have used virtual planning for making surgical guides in different ways to aid in component positioning. Buller et al. used CT scan based three dimensional pre-operative planning software for designing patientspecific implant and instrumentation (PSI) and reported increased precision in comparison to standard technique. However, the study was done on saw bone and hence lacked consideration of factors like exposure and soft tissues. Sakai et al. conducted a cadaveric study to evaluate patient-specific surgical guides for neck cut and cup positioning [24]. Travis et al. [20] also reported significantly greater accuracy using 3D preoperative planning along with PSI than traditional planning and instrumentation.

In our study, 3D printed jigs were of low cost (4-6 USD each) and apart from designing the jigs, we also determined cup size on 3D model, which matched with intra-operative cup size in all the 18 hips of group-A. The virtual planning also helped us in knowing the altered anatomy like bone loss, locations of osteophytes, depth of acetabulum etc. and hence made us better prepared for surgeries. The acetabular jigs designed in our study for group-A patients were patient specific. Although the placement of jig required a bit more exposure on postero-superior region of acetabulum, its placement was an easy task. Acetabular jig composed of PLA (polylactic acid) can be sterilized by many methods including heat sterilization method either with saturated steam at $125{ }^{\circ} \mathrm{C}$ to $130{ }^{\circ} \mathrm{C}$ for around $20 \mathrm{~min}$ or hot air at $160{ }^{\circ} \mathrm{C}$ for $2 \mathrm{~h}$, respectively. Other methods used like gamma irradiation, UV irradiation, ETO (ethylene oxide) are also used but are known to change the property of material. Under standard autoclave, PLA tends to be more brittle rather than deformed and its brittleness increases with number of time it is autoclaved, as experienced in many other studies. We used standard autoclave method and experienced the same. But autoclaving PLA in our study had no deterrent effect in our study as we used it as a guiding jig and was not exposed to any external forces.

The difference in blood loss and surgical duration was statistically insignificant indicating that the jig can be used with only minimal increase in duration and blood loss. Flordal et al. studied 212 patients undergone total hip replacement surgery and estimated average surgical duration of 89 min [25]. Despite of a good subjective impression of the virtual method, the statistical significance was not reached with respect to the accuracy of cup placement. This may be attributed to the few limitations of our study including small sample size, diverse indications and inhomogeneous level of complexity in both groups. This technique may not be useful in bilateral cases where acetabular landmarks have been distorted. But in cases like bilateral avascular necrosis of femoral head where acetabular landmarks are not distorted, this technique can be useful in bilateral cases. During the course of our study there were some modifications in design of acetabular jig which were mainly of the attachment part which only limited the exposure. Guiding arc of this jig was not changed hence the results of our study were not supposed to be affected by these variations. Although a study with a large sample size with our final jig design is required to obtain more significant results.

The mean angle of anteversion and inclination in both groups in our study lied in safe range. However, the values of the angle of anteversion in group-A were noted to have less variability with less outlier than group-B suggesting a clinical advantage of the jig in guiding cup placement. The jig was particularly more useful in conditions where the anatomical landmarks were distorted like in malunited acetabular fractures and bony ankylosis of hip.

Our initial experience with the use of virtual planning for patient-specific 3D printed jig has been promising. We believe a large sample size study is warranted for validation of our study.

\section{Conclusions}

3D printed acetabular jig were found to match the surface of patient's pelvic bone precisely without increasing surgical morbidity. Acetabular cup placement under the guidance of 
acetabular jig maintains angle of inclination and anteversion within safe zone recommended for maximum hip stability and keeps centre of rotation closer to original hip rotation centre. However, a large homogenous sample size study is warranted to further validate our initial experience with this jig.

Acknowledgements This study has not received any funds/grants from any individual or organization. All procedures performed in studies involving human participants were in accordance with the ethical standards of the institutional and committee and with the 1964 Helsinki declaration and its later amendments or comparable ethical standards.

\section{Compliance with Ethical Standards}

Conflict of interest On behalf of all authors, the corresponding author states that there is no conflict of interest.

Ethical standard statement The authors certify that they have obtained all appropriate patient consent forms. In the form the patient(s) has/ have given his/her/their consent for his/her/their images and other clinical information to be reported in the journal. The patients understand that their names and initials will not be published and due efforts will be made to conceal their identity, but anonymity cannot be guaranteed."

Informed consent Informed consent of each patient has been taken individually for undergoing this study.

\section{References}

1. von Knoch, M., Berry, D. J., Harmsen, W. S., \& Morrey, B. F. (2002). Late dislocation after total hip arthroplasty. Journal of Bone and Joint Surgery American, 84(11), 1949-1953.

2. Kennedy, J. G., Rogers, W. B., Soffe, K. E., Sullivan, R. J., Griffen, D. G., \& Sheehan, L. J. (1998). Effect of acetabular component orientation on recurrent dislocation, pelvic osteolysis, polyethylene wear, and component migration. Journal of Arthroplasty, 13(5), 530-534.

3. Lewinnek, G. E., Lewis, J. L., Tarr, R., Compere, C. L., \& Zimmerman, J. R. (1978). Dislocations after total hip replacement arthroplasties. Journal of Bone and Joint Surgery American, 60(2), 217-220.

4. Dorr, L. D., Wolf, A. W., Chandler, R., \& Conaty, J. P. (1983). Classification and treatment of dislocations of total hip arthroplasty. Clinical Orthopaedics and Related Research, 173, 151-158.

5. Nolte, L. P., \& Beutler, T. (2004). Basic principle of CAOS. Injury, 351, 6-16.

6. Callanan, M. C., Jarrett, B., Bragdon, C. R., Zurakowski, D., Rubash, H. E., Freiberg, A. A., et al. (2011). The John Charnley Award: risk factors for cup malpositioning: quality improvement through joint registry at a tertiary hospital. Clinical Orthopaedics and Related Research, 469(2), 319-329.

7. McCollum, D. E., \& Gray, W. J. (1990). Dislocation after total hip arthroplasty. Causes and prevention. Clinical Orthopaedics and Related Research, 261, 159-170.

8. Scheerlinck, Thierry. (2014). Cup positioning in total hip arthroplasty. Acta Orthopaedica Belgica, 80(3), 336-347.

9. Pradhan, Riten. (1999). Planar anteversion of the acetabular cup as determined from plain anteroposterior radiographs. Journal of Bone and Joint Surgery British, 81(3), 431-435.
10. Alberton, G. M., \& HighWA, Morrey B. F. (2002). Dislocation after revision total hip arthroplasty: an analysis of risk factors and treatment options. Journal of Bone and Joint Surgery American, 84(10), 1788-1792.

11. Phillips, C. B., Barrett, J. A., Losina, E., Mahomed, N. N., Lingard, E. A., Guadagnoli, E., et al. (2003). Incidence rates of dislocation, pulmonary embolism, and deep infection during the first six months after elective total hip replacement. Journal of Bone and Joint Surgery American, 85(1), 20-26.

12. Parvizi, J., Wade, F. A., Rapuri, V., Springer, B. D., Berry, D. J., \& Hozack, W. J. (2006). Revision hip arthroplasty for late instability secondary to polyethylene wear. Clinical Orthopaedics and Related Research, 447, 66-69.

13. Robinson, R. P., Simonian, P. T., Gradisar, I. M., \& Ching, R. P. (1997). Joint motion and surface contact area related to component position in total hip arthroplasty. Journal of Bone and Joint Surgery British, 79(1), 140-146.

14. Wera, G. D., Ting, N. T., Moric, M., Paprosky, W. G., Sporer, S. M., \& Della Valle, C. J. (2012). Classification and management of the unstable total hip arthroplasty. Journal of Arthroplasty, 27(5), $710-715$.

15. Jolles, B. M., Zangger, P., \& Leyvraz, P. F. (2002). Factors predisposing to dislocation after primary total hip prosthesis. Journal of Arthroplasty, 17(3), 282-288.

16. Ryan, J. A., Jamali, A. A., \& Bargar, W. L. (2010). Accuracy of computer navigation for acetabular component placement in THA. Clinical Orthopaedics and Related Research, 468(1), 169-177.

17. Sendtner, E., Schuster, T., Wörner, M., Kalteis, T., Grifka, J., \& Renkawitz, T. (2011). Accuracy of acetabular cup placement in computer-assisted, minimally-invasive THR in a lateral decubitus position. International Orthopaedics, 35(6), 809-815.

18. Tay, X. W., Zhang, B. X., \& Gayagay, G. (2017). Use of iPhone technology in improving acetabular component position in total hip arthroplasty. Arthroplast Today, 3(3), 167-170.

19. Sotereanos, N. G., Mark, C. M., Brett, S., Robert, H., Jeffrey, J. S., \& David, W. (2006). Using intraoperative pelvic landmarks for acetabular component placement in total hip arthroplasty. Journal of Arthroplasty, 21(6), 832-840.

20. Small, T. K., Molloy, R., Bryan, J., Klika, A. K., \& Barsoum, W. K. (2014). Comparison of acetabular shell position using patient specific instruments vs. standard surgical instruments: a randomized clinical trial. Journal of Arthroplasty, 29(5), 1030-1037.

21. Murray, D. W. (1993). The definition and measurement of acetabular orientation. Journal of Bone and Joint Surgery British, 75(2), 228-232.

22. Ali, K. M. A., Brakenbury, P. H., \& Reynolds, I. S. (1981). Dislocation following total hip replacement. Journal of Bone and Joint Surgery British, 63(2), 214-218.

23. Patel, A. B., Wagle, R. R., Usrey, M. M., Thompson, M. T., Incavo, S. J., \& Noble, P. C. (2010). Guidelines for implant placement to minimize impingement during activities of daily living after total hip arthroplasty. Journal of Arthroplasty, 25(8), $1275-1281$.

24. Sakai, T., Hanada, T., Murase, T., Kitada, M., Hamada, H., Yoshikawa, H., et al. (2014). Validation of patient specific surgical guides in total hip arthroplasty. The International Journal of Medical Robotics, 10(1), 113-120.

25. Flordal, P. A., \& Neander, G. (1991). Blood loss in total hip replacement. Archives of Orthopaedic and Trauma Surgery, 111(1), 34-38.

Publisher's Note Springer Nature remains neutral with regard to jurisdictional claims in published maps and institutional affiliations. 